\title{
An alternative method for deriving a USLE nomograph $K$ factor equation
}

\author{
S.J. Walker $^{\mathrm{a}}$ \\ ${ }^{a}$ Centre for Water and Landscape Dynamics, The Fenner School of Environment and Society, The Australian \\ National University, Australian Capital Territory \\ Email:simon.walker@anu.edu.au
}

\begin{abstract}
Soil erodibility (K factor) is an important component of the Universal Soil Loss Equation (USLE) / Revised USLE (RUSLE) and is used in many erosion models. A soil erodibility nomograph developed by Wischmeier et al. (1971) allows for a simple and repeatable method for calculating a $\mathrm{K}$ factor based on five key soil attributes. These attributes include the percentage of silt + very fine sand, sand and organic matter to calculate a first approximation of $\mathrm{K}$. A value for soil structure and a value for permeability can then be used to complete the nomograph where a first approximation is insufficient.
\end{abstract}

Following the development of the nomograph, an equation was created to bypass the otherwise tedious and time consuming manual process. This classical soil $\mathrm{K}$ factor equation can emulate the nomograph with some accuracy, but has shown to lack the required precision in certain circumstances. In this research a new method for deriving an equation directly from the soil erodibility nomograph is demonstrated and tested against the classical $\mathrm{K}$ factor equation.

The main aims of this work are to:

1. Test a method for deriving a $\mathrm{K}$ factor equation that facilitates graphical modification to the nomograph prior to generating an equation.

2. Compare the results against those achieved using the classical $\mathrm{K}$ factor equation.

3. Create resources (including open-source scripts and a custom toolbox for ESRI ArcGIS) to allow ease of access to these methods for researchers and landscape managers alike.

The method assumes that by segmenting the nomograph into zones according to the percentage of silt + fine sand, the relationship between key soil properties and erodibility can then be considered as a series of linear equations and can hence be solved within a linear systems framework.

This method allows for iterative creation of new equations to fit any graphical changes made to the nomograph, and results show it can produce equations that emulate the nomograph solution with higher accuracy than the classical $\mathrm{K}$ factor equation.

When tested on 100 randomly generated theoretical soil samples, this method achieves an average difference of just $25 \%$ from the nomograph solution. The classical equation achieves an average difference of $38 \%$ from the nomograph solution when tested on the same set of theoretical soil samples.

Furthermore, where the classical equation is limited to soil samples with $<70 \%$ silt + fine sand, this new method can solve the full range of values taken by the nomograph and does not require additional equations for edge cases.

This work shows that alternatives methods can solve the soil erodibility nomograph with higher accuracy than the classical equation, and the method is not restricted to the soil erodibility nomograph, it can also be applied to other biophysical nomographs.

A custom toolbox is developed for ESRI ArcGIS software to allow use of the equations developed here without the need to replicate the methods. A range of open-source scripts written in the Python programming language are also available.

Keywords: $\quad$ Universal Soil Loss Equation (USLE), soil erodibility nomograph, K factor equation 


\section{INTRODUCTION}

The soil erodibility factor ( $\mathrm{K}$ factor) is a key component of the Universal Soil Loss Equation (USLE) / Revised USLE (RUSLE) and is used in many erosion models (Auerswald et al., 2014). A soil erodibility nomograph developed by Wischmeier et al. (1971) allows for a simple and repeatable method for calculating a K factor based on five key soil attributes. These attributes include the percentage of silt + very fine sand, sand and organic matter to calculate a first approximation of $\mathrm{K}$. A value for soil structure and a value for permeability can then be incorporated to complete the nomograph where a first approximation is insufficient. Later, Wischmeier and Smith (1978) developed the widely used K factor equation (Equation 1) to allow quick and easy calculation of soil erodibility without the need to follow the nomograph.

$100 K=2.1 * M^{1.14} *\left(10^{-4}\right) *(12-a)+3.25(b-2)+2.5(c-3)$

Where $M$ is (percentage of silt + very fine sand $) *(100-$ percent clay $), a$ is the percentage of organic matter, $b$ is a soil structure class and $c$ is profile-permeability class (Wischmeier and Smith, 1978).

Although various forms of the equation have been developed over the years, the original equation is still commonly used by modern studies, for example Panagos et al. (2014) and Teng et al. (2016), for estimating a soil $\mathrm{K}$ value without the nomograph.

While the soil erodibility nomograph can be emulated reasonably well with the classical $\mathrm{K}$ factor equation, there are scenarios in which it is unable to work effectively. For example, the equation cannot compute a $\mathrm{K}$ value for soils with $>70 \%$ silt and fine sand (Auerswald et al., 2014). For this specific case, the curves on the nomograph are drawn to include an inflection point at the $70 \%$ silt + fine sand mark (Wischmeier and Smith, 1978).

Later studies have found that, in some environments, systematic differences exist between nomograph-derived $\mathrm{K}$ values and observed K values (Zhang et al., 2008, Panagos et al., 2014), and that such discrepancies can be adjusted for with the introduction of additional variables. However, adjusting the classical equation to account for additional variables is difficult, time consuming, and requires knowledge of the processes used to derive the original equation.

Accordingly, the main aims of this work are to:

1. Test a method for deriving a $\mathrm{K}$ factor equation that facilitates graphical modification to the nomograph prior to generating a set of equations.

2. Compare the results against those achieved using the classical $\mathrm{K}$ factor equation.

3. Create resources (including open-source scripts and a custom toolbox for ESRI ArcGIS) to allow ease of access to these methods for researchers and landscape managers alike.

\section{METHODS}

The methods used in this paper represent an entirely new way of deriving a $\mathrm{K}$ factor equation directly from the soil nomograph in a way that allows graphical changes to be made to the nomograph prior to resolving a new set of equations. The method assumes that by segmenting the nomograph according to the percentage of silt + fine sand, the relationship between key soil properties and erodibility can then be considered as a series of linear equations, and can hence be solved within a linear systems framework. The specific method used in this study is a form of Gaussian elimination known as Gauss-Jordan Reduction (Althoen and Mclaughlin, 1987).

Gaussian elimination, and the related Gauss-Jordan Reduction, are methods for finding the best linear function to approximate some set of observations. In this work, Gauss-Jordan Reduction is used as a simple and direct method for deriving the best set of linear functions for solving the soil erodibility nomograph. 


\subsection{Linearization framework}

If an assumption of linearity is made, then each combination of three soil attributes and the resultant $\mathrm{K}$ factor can be described by the linear equation:

$X_{i}+Y_{i}+Z_{i}-K_{i}=0$

The nomograph can then be manually solved three separate times and the values inserted into Equation 2 such that, for sample $i$, $X_{i}$ equals the percent silt + fine sand, $Y_{i}$ equals the percent sand, $Z_{i}$ equals the percent organic matter and $K_{i}$ equals the first approximation of $\mathrm{K}$. It is then possible to reorganise the three resulting equations into a system of simultaneous linear equations:

$\left\{\begin{array}{lll}X_{1} & Y_{1} & Z_{1}=K_{1} \\ X_{2} & Y_{2} & Z_{2}=K_{2} \\ X_{3} & Y_{3} & Z_{3}=K_{3}\end{array}\right.$

Once the equations have been organised into matrix form, Gauss-Jordan Reduction can be performed to convert the matrix into reduced row echelon form and solved for a unique solution. The example soil samples (red lines) in Figure 1 produce the following $3 \times 3$ system in augmented matrix form:

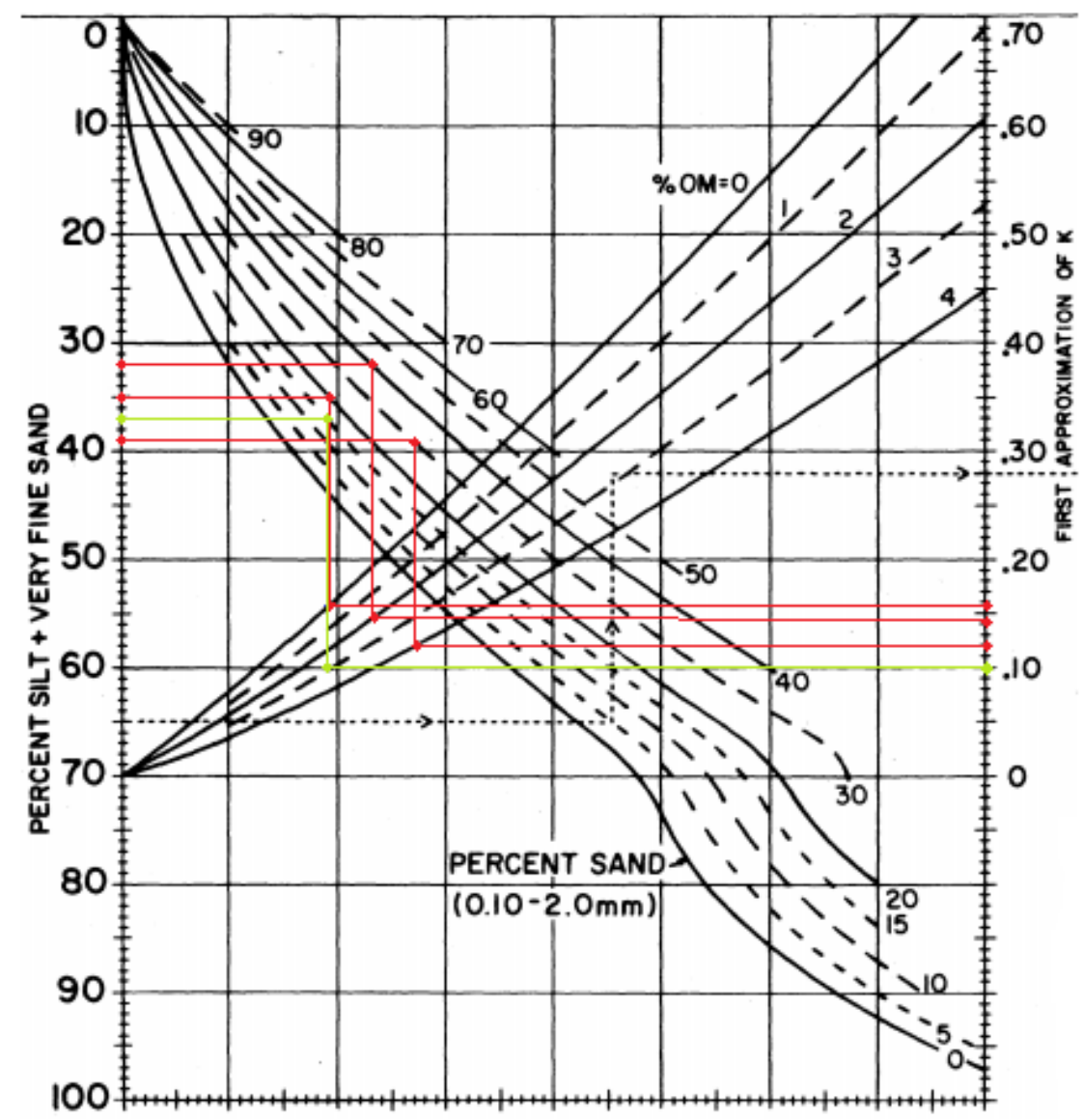

Figure 1. Example process of setting up a linear system from values lying between $30-40 \%$ silt + fine sand. Three simultaneous linear equations are generated according to values taken for each soil attribute. Figure adapted from (Wischmeier and Smith, 1978).

$\left[\begin{array}{llll}32 & 40 & 2 & 0.14 \\ 35 & 20 & 0 & 0.16 \\ 39 & 30 & 4 & 0.12\end{array}\right]$

Converting the above matrix into reduced row echelon form following Gauss-Jordan Reduction yields the unique solution $X=0.0038, Y=0.0013$ and $Z=-0.017$.

The coefficients for $X, Y$ and $Z$, described by the unique solution, can then be substituted into a new equation:

$a_{i} X+b_{i} Y+c_{i} Z=K_{i}$

Where, for a given soil sample $i, a_{i}$ is the percentage silt + fine sand, $b_{i}$ is the percentage sand and $c_{i}$ is the percentage organic matter.

The green line on Figure 1 shows the nomograph solution for a fourth combination of values between the range $30-40 \%$ silt + fine sand ( silt + fine sand $=37$, sand $=15$, and organic matter $=3$ ).

By solving this combination using the nomograph, the resulting first approximation of $\mathrm{K}$ is equal to 0.10 . When the same values for silt + fine sand, sand and organic matter are substituted into Equation 5, the resulting K value is 0.11 . The difference between the nomograph solution and the solution found using the linearization method in this example is 0.01 . 
In cases where a first approximation of $\mathrm{K}$ is not sufficient, the process can be repeated starting from Equation 2 and using the first approximation of $\mathrm{K}$ from the first run as the $X_{i}$ variable, soil structure as the $Y_{i}$ variable and soil permeability as the $Z_{i}$ variable (Figure 2). For Equation $5, a_{i}, b_{i}$ and $c_{i}$ then become the first approximation of $\mathrm{K}$, soil structure and soil permeability, respectively. This allows the process to solve the full nomograph as would be the case using the classical equation. In the example case from Figure 2, the 3 $\times 3$ augmented matrix contains:

$\left[\begin{array}{llll}0.14 & 2 & 3 & 0.14 \\ 0.16 & 4 & 1 & 0.18 \\ 0.12 & 1 & 5 & 0.15\end{array}\right]$

The resulting coefficients from this are $X=-0.2941, Y=0.05$ and $Z=0.0271$.

Again, a theoretical soil sample (green line, Figure 2) is taken and solved using the nomograph, resulting in a $\mathrm{K}$ value of 0.21 . The $X, Y$ and $Z$ values for this test sample are then substituted into Equation 5, yielding a predicted $\mathrm{K}$ value of 0.21

\subsection{Testing against classical equation}

To test the performance of this new method against that of the classical

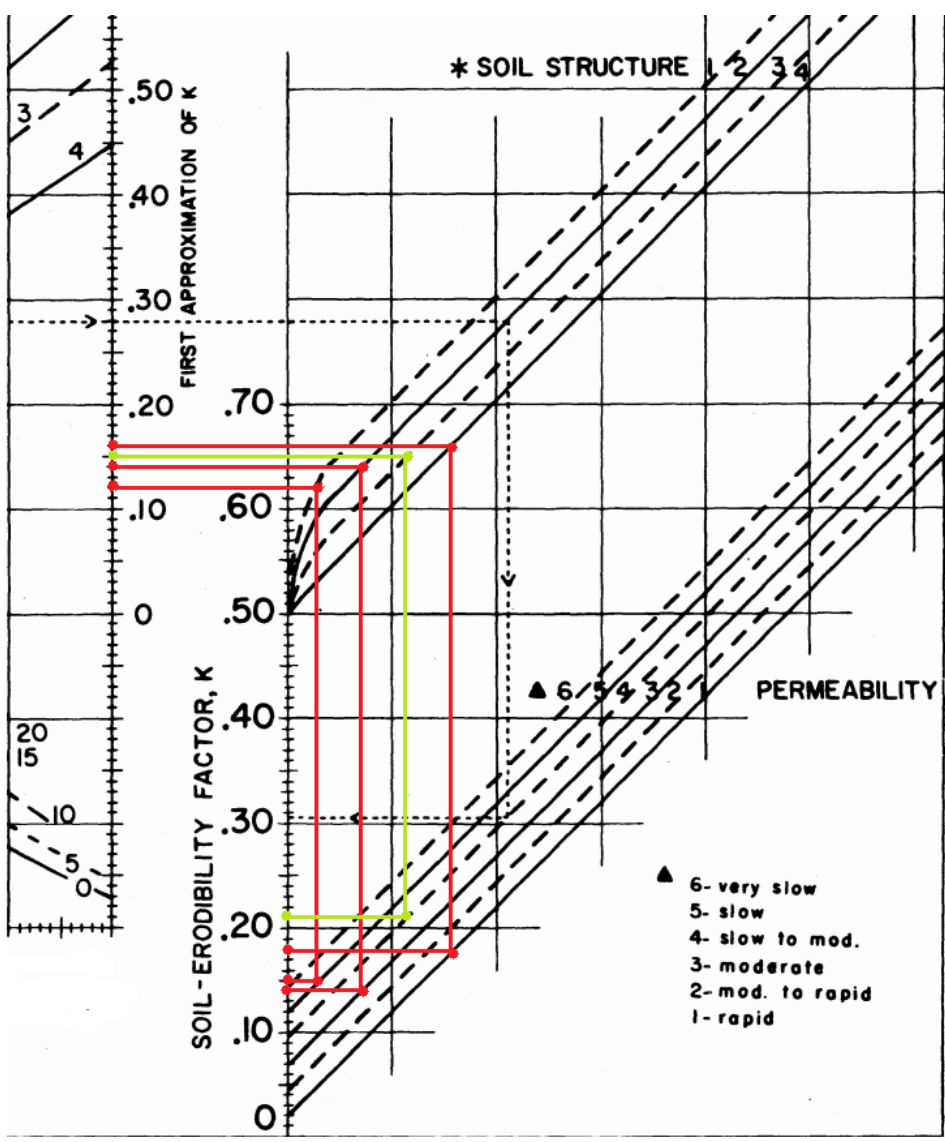

Figure 2. Example of repeat process using the first approximation from Figure 1 as the $X$ variable, soil structure as the $Y$ variable and soil permeability as the $Z$ variable. Figure adapted from

(Wischmeier and Smith, 1978).

equation, 100 randomly generated theoretical soil samples were first created. To facilitate assessment of values across the full range of possible inputs, the nomograph was divided into 14 evenly spaced horizontal segments each containing a possible initial silt + fine sand percentage between a narrow $5 \%$ range. For example, the first seven random samples taken all had a silt + fine sand content somewhere between $0-5 \%$, with values for sand and organic matter taking on random values within the range of the nomograph.

$\mathrm{K}$ was then computed for each randomly generated soil sample by manually solving the nomograph. The resulting values were taken to be validation data against which outputs from the classical equation and from the linearization method were measured. All values were first converted to SI units ( $\left.\mathrm{t} \mathrm{ha} \mathrm{h} \mathrm{MJ} \mathrm{Ma}^{-1} \mathrm{ham}^{-1}\right) \mathrm{using}$ a conversion factor of 1.313 specified by Wischmeier and Smith (1978).

\subsection{Development of a custom toolbox}

The workflow outlined in section 2.1 was integrated into a model designed for ESRI ArcGIS software and packaged into a custom toolbox. This allows researchers and landscape managers to use this method with gridded soil attribute datasets without the need to go through any of the steps outlined here. The workflow was also written as a series programs in the open-source Python programming language (https://github.com/SimonJW/erosion).

\section{RESULTS}

\subsection{Overview}

Overall, the method outlined in this paper performs better than the classical $\mathrm{K}$ factor equation when assessed as an average percentage difference from the nomograph solution. However, when assessed as an average difference in $\mathrm{K}$ value, the two perform equally well (Table 1). 
Table 1. Comparison of average performance between the classical equation and the linearization method. Values represent the difference between the $\mathrm{K}$ value derived from the nomograph, and values predicted by the classical equation and the linearization method (error represent standard error).

\section{Average performance}

Percent difference from nomograph

Percent difference from nomograph excluding five highest values

$\mathrm{K}$ value difference ( $\mathrm{t}$ ha h $\mathrm{MJ}^{-1} \mathrm{ha}^{-1} \mathrm{~cm}^{-1}$ )

$\mathrm{K}$ value difference ( $\mathrm{t}$ ha h $\mathrm{MJ}^{-1} \mathrm{ha}^{-1} \mathrm{~cm}^{-1}$ ) excluding five highest values

\section{Linearization method Classical equation}

\begin{tabular}{|l|l|}
\hline $25.4 \pm 3.4$ & $38 \pm 6.8$ \\
\hline $24.5 \pm 3.5$ & $32.8 \pm 6.0$ \\
\hline $0.037 \pm 0.0038$ & $0.037 \pm 0.0028$ \\
\hline $0.030 \pm 0.0025$ & $0.032 \pm 0.0021$ \\
\hline
\end{tabular}

To illustrate where differences are occurring between the two methods, results of each are plotted against the silt + fine sand fraction in Figure 3. This shows the tendency of the classical equation to underperform in the range of $0-10 \%$ silt + fine sand fraction, and the linearization method to underperform in the range $50-60 \%$. The zone in which the classical equation is underperforming is also the zone of steepest curve on the nomograph. This indicates that the ability of the classical equation to accurately emulate the nomograph may be negatively impacted by this high curve steepness.

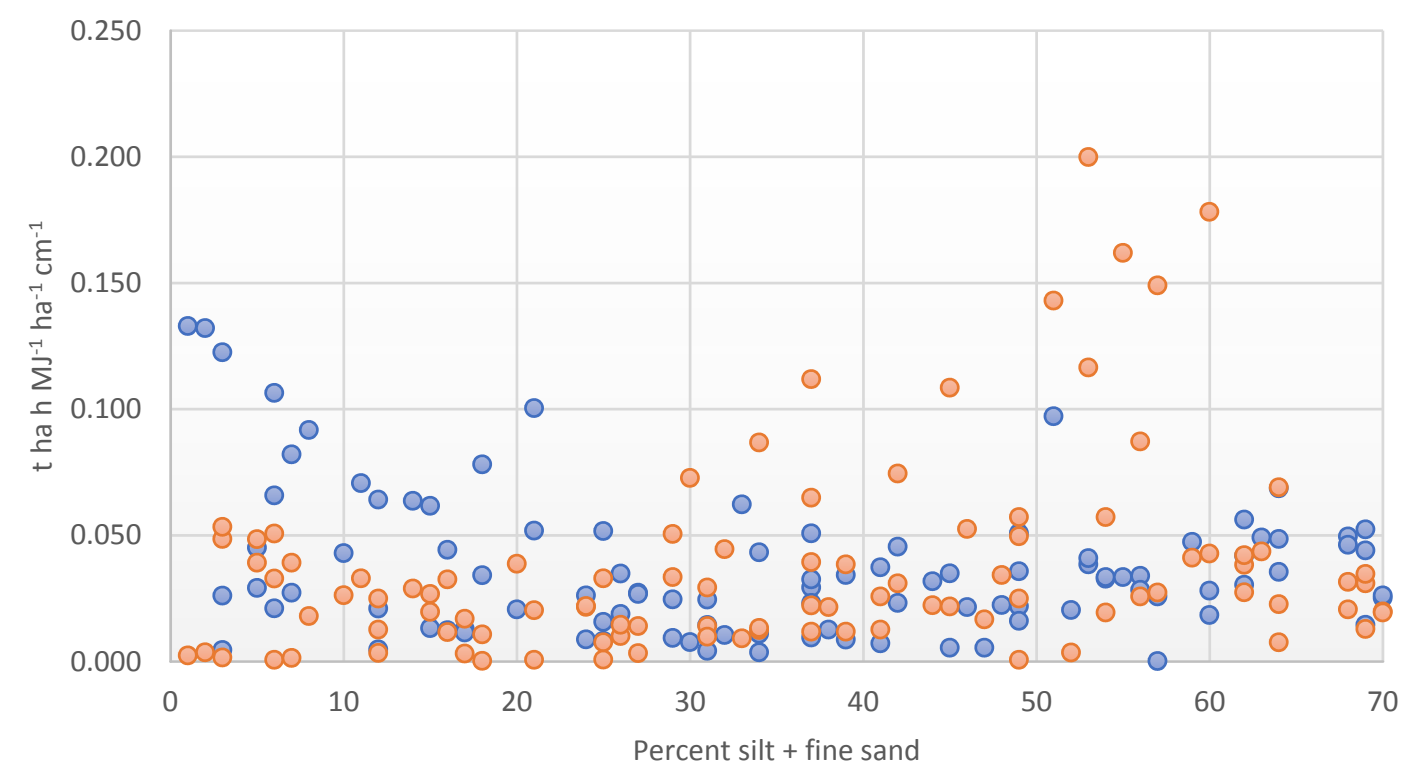

O Classical equation O Linearization method

Figure 3. Plot showing $K$ values calculated using the classical equation (blue) and the linearization method (orange) subtracted from $\mathrm{K}$ values derived using the nomograph (i.e. the difference between predicted and observed). Units have been converted to metric system SI units using the conversion coefficient specified by (Wischmeier and Smith, 1978).

If the five values furthest from the nomograph solution are excluded from each dataset, the linearization method improves in average accuracy by $0.007 \mathrm{t}$ ha h MJ $\mathrm{MJ}^{-1} \mathrm{ha}^{-1} \mathrm{~cm}^{-1}$, while the classical equation improves by $0.005 \mathrm{t}$ ha $\mathrm{h} \mathrm{MJ}^{-1} \mathrm{ha}^{-1} \mathrm{~cm}^{-1}$. However, these improvements may be small relative to the error contained in many input data to a GIS model.

Although the difference in performance between the two methods is negligible with respect to average difference in the physical units $\mathrm{t}$ ha $\mathrm{h} \mathrm{MJ}^{-1} \mathrm{ha}^{-1} \mathrm{~cm}^{-1}$, the performance of the linearization method is notably better when considering the difference from the nomograph solution as a percentage (Figure 4). 


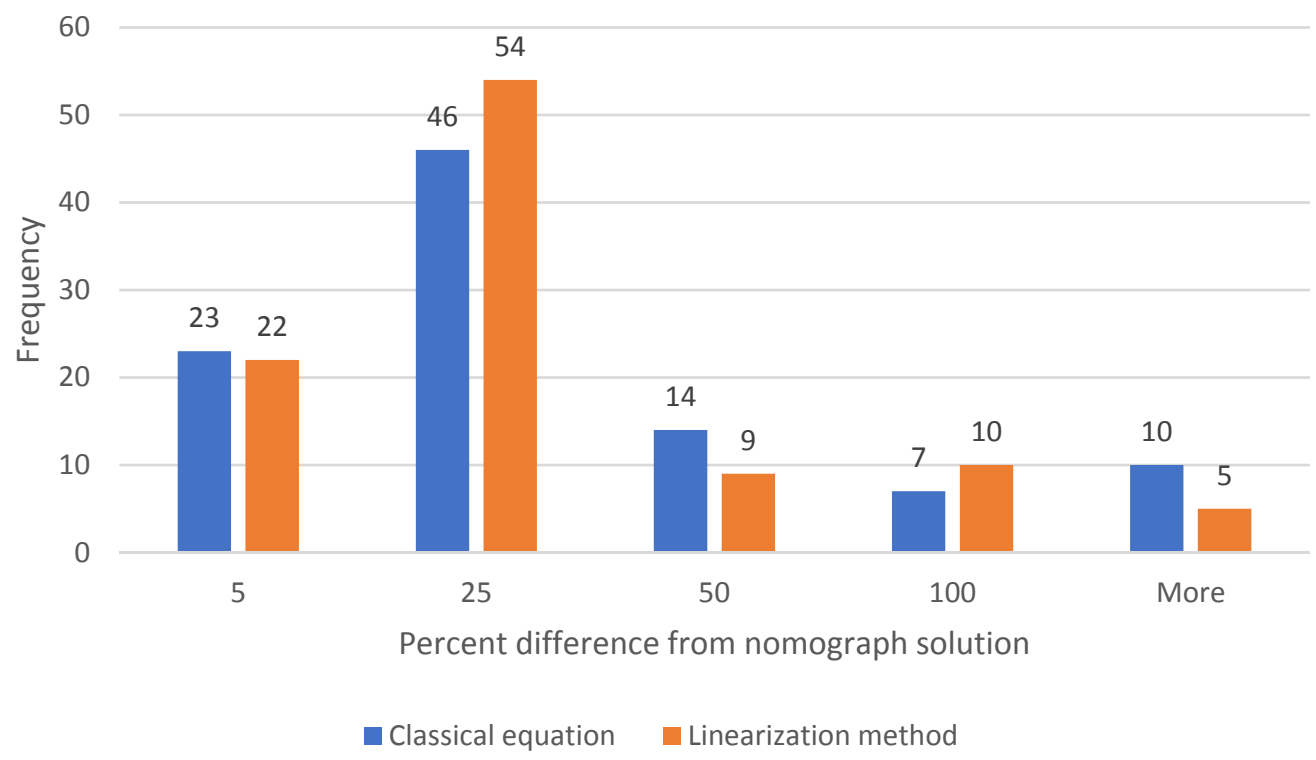

Figure 4. Frequency distribution histogram showing difference in percentage between the nomographderived $\mathrm{K}$ values and $\mathrm{K}$ values predicted by both the classical equation (blue) and the linearization method (orange). Values on the $x$-axis represent maximum values for a give data bin (with the corresponding minimum being $1+$ the maximum of the previous bin).

\section{DISCUSSION AND CONCLUSIONS}

\subsection{Overview}

The findings here support previous studies on the nature of $\mathrm{K}$ value observations across different environments. For example, Renard et al. (1997) found that the best method for determining an appropriate K factor depends on the environment, and the nomograph developed by (Wischmeier et al., 1971) is only suitable for particular settings. Similarly, Zhang et al. (2008) found that systematic differences between K values computed by the nomograph and those observed across varying environments. They suggest that the regular nature of the variation lends itself to adjustment with linear regression analysis. Systematic differences are also apparent in the results of this current study. For example, the linearization method shows consistent difficulty predicting $\mathrm{K}$ values for soils with a silt + fine sand percentage between $50-60 \%$. Meanwhile the classical $\mathrm{K}$ factor equation showed similar concentrated error for soils with a silt + fine sand percentage between $0-10 \%$.

\subsection{Flexibility of the linearization method}

The results for this study show that applying a linearization method to derive equations directly from the soil nomograph has several advantages over using the classical equation. The classical equation developed to emulate the nomograph does not cope with the full range of values, and the nomograph must be used in cases where the equation is unsuitable (Auerswald et al., 2014). The linearization method outlined in this paper is not limited in this same manner, and can generate equations solving the full nomograph. Furthermore, it also facilitates pre-calculation graphical changes to the nomograph. For example, Auerswald et al. (2014) found that the average organic content of arable top soils in their study was $3.5 \%$, meaning the average value is already almost at the upper limit of what the soil nomograph can accept. Hence if some clear effect of higher organic matter content is apparent, then it may be desirable to extend the range of organic matter contents accepted by the nomograph to $5 \%$ or $6 \%$, rather than being limited to a maximum of $4 \%$. By using the linearization method described here, new lines can be added to the nomograph and the subsequent process of deriving equations to emulate the new nomograph remains the same.

The shape of curves can also be edited, or new variables added and the process does not change. Allowing for the addition of extra variables would help address issues such as the propensity for the nomograph to overestimate erosion in Mediterranean soils unless surface stone cover is accounted for (Panagos et al., 2014). Similarly, Mutchler and Carter (1983) found that seasonal effects can change observed K values by factor of five for a given area. The method outlined here could incorporate appropriate seasonal curves prior to calculation of equations. 


\subsection{Further work}

Further work should examine whether the zone in which predicted $\mathrm{K}$ values are furthest from the nomograph solution (i.e. $50-60 \%$ silt + fine sand) are likely to occur more often in specific environments, and whether the introduction of additional variables (or expansion of the range of current variable limits) can help to address this. For example, the nomograph is generally better suited to less aggregated soils and many studies have found that average aggregate size or some form of aggregation index provides the most important addition variable (Renard et al., 1997).

Also, given the underlying assumption of linearity, it is expected that this method would perform better if narrower bands are used in the initial derivation of a set of linear equations. It would be beneficial to test whether this is indeed the case, and assess the degree of improvement gained by further segmentation of the nomograph.

Finally, the nature of this method is such that it is not limited to the soil erodibility nomograph. In theory, this method can be applied to any nomograph representing a physical process. Further work should apply this method to other systems and test its ability to produce reliable predictive linear equations.

\section{ACKNOWLEDGMENTS}

I would like to acknowledge Professor Albert van Dijk and Dr Bruce Doran for their encouragement and suggestions towards improving this work.

\section{REFERENCES}

Althoen, S. C. \& Mclaughlin, R. (1987). Gauss-Jordan reduction: A brief history. The American mathematical monthly, 94, 130-142.

Auerswald, K., Fiener, P., Martin, W. \& Elhaus, D. (2014). Use and misuse of the K factor equation in soil erosion modeling: An alternative equation for determining USLE nomograph soil erodibility values. CATENA, 118, 220-225.

Mutchler, C. K. \& Carter, C. E. (1983). Soil erodibility variation during the year. Transactions of the ASAE, 26, 1102-1104.

Panagos, P., Meusburger, K., Ballabio, C., Borrelli, P. \& Alewell, C. (2014). Soil erodibility in Europe: A highresolution dataset based on LUCAS. Science of The Total Environment, 479, 189-200.

Renard, K., Meyer, L. \& Foster, G. (1997). INTRODUCTION AND HISTORY. Predicting soil erosion by water: a guide to conservation planning with the revised universal soil loss equation (RUSLE), 1.

Teng, H., Viscarra Rossel, R. A., Shi, Z., Behrens, T., Chappell, A. \& Bui, E. (2016.) Assimilating satellite imagery and visible-near infrared spectroscopy to model and map soil loss by water erosion in Australia. Environmental Modelling \& Software, 77, 156-167.

Wischmeier, W. H., Johnson, C. B. \& Cross, B. V. (1971). A soil erodibility nomograph for farmland and construction sites. Journal of soil and water conservation, 26, 189-193.

Wischmeier, W. H. \& Smith, D. D. (1978). Predicting rainfall erosion losses-a guide to conservation planning. Predicting rainfall erosion losses-a guide to conservation planning.

Zhang, K. L., Shu, A. P., Xu, X. L., Yang, Q. K. \& Yu, B. (2008). Soil erodibility and its estimation for agricultural soils in China. Journal of Arid Environments, 72, 1002-1011. 